\title{
Prevalence of peripheral arterial diseases in patients with large artery ischemic stroke and its prognostic value
}

\author{
Sherien Farag ${ }^{1}$, Mahmoud Elbalkimy $^{1}$, Ahmed Elbassiouny $^{1}$, John George ${ }^{2}$ and Mai Fathy ${ }^{1 *}$ (D)
}

\begin{abstract}
Background: Peripheral artery disease (PAD) can be an important predictor of comorbid cerebrovascular disease (CVD). However, it is not sufficiently investigated or considered in the management and prevention of stroke.

Objectives: To study the prevalence of peripheral arterial disease in large artery ischemic stroke patients and its impact on prognosis.

Methods: This is a prospective cohort study. A total of 100 patients with large artery ischemic stroke were enrolled with assessment for signs of PAD; duplex on both lower limb arteries and measurement of ankle-brachial index $(\mathrm{ABI})$, carotid duplex, and/or C.T angiography brain and neck and/or conventional angiography; functional assessment by NIHSS at days 0, 7, and 30; and follow-up for recurrence or death after 6 months.

Results: Peripheral arterial disease was found in $42 \%$ of cases. Fifty percent of patients with lower extremity arterial disease were asymptomatic. Fifty percent had significant carotid stenosis (stenosis $>50 \%$ ), and $48 \%$ of patients with significant carotid stenosis had peripheral arterial disease. During our study, 8 cases had a recurrence of large artery ischemic stroke, and 5 cases (62.5\%) had PAD. The recurrence rate of stroke was the highest in the asymptomatic PAD group with $19 \%$.

Conclusion: The prevalence of PAD in large artery ischemic stroke patients is high. Asymptomatic PAD has a risk potential for large artery ischemic stroke recurrence and mortality more than symptomatic PAD and non-PAD patients.
\end{abstract}

Keywords: Peripheral artery disease, Ankle-brachial index, Stroke outcome

\section{Introduction}

The presence of vascular disease in one arterial territory strongly predicts disease in other territories, increasing the risk of vascular events in patients with previous stroke/transient ischemic attack [1].

$\mathrm{PAD}$ can be either asymptomatic (defined by an $\mathrm{ABI} \leq$ 0.9 ) or symptomatic presented by intermittent claudication or acute PAD events (acute limb ischemia and critical limb ischemia). Acute limb ischemia can be viable,

\footnotetext{
* Correspondence: maifathy23@gmail.com

${ }^{1}$ Neurology Department, Faculty of Medicine, Ain Shams University, Cairo, Egypt

Full list of author information is available at the end of the article
}

threatened, or irreversible. Critical limb ischemia includes rest pain and ulceration [2].

$\mathrm{ABI}$ is an easy way to compare the systolic pressure of the upper extremity with that of the affected lower extremity. ABI test has a sensitivity above $90 \%$ and a specificity of $95 \%$ for the diagnosis of PAD [3].

Although PAD risks poor prognosis compared with vascular disease in other territories, little attention is paid to its epidemiology, treatment, and prevention. Despite the high prevalence of PAD in patients with stroke, and of stroke in patients with PAD, PAD is not considered in the guidelines for treatment, prevention, and rehabilitation of stroke. Therefore, screening for PAD is 
not routinely considered, and so, it is probably often missed [4].

PAD affects 12 to $14 \%$ of the general population. The prevalence of PAD is age-dependent, reaching 10\% in patients aged over 60 years. Unfortunately, the majority of patients are asymptomatic and undiagnosed. Also, one third of patients with symptoms do not report them to their doctor. As a result of this, a high-risk group of patients is underdiagnosed and receive no (or suboptimal) treatment or intervention [5].

The prevalence of asymptomatic carotid stenosis increases with decreasing ABI. Therefore, there may be a role for screening for asymptomatic carotid stenosis in patients with PAD, although the benefit of carotid endarterectomy in such patients is low [6].

\section{Aim of this work}

The aim of this work is to study the prevalence of peripheral arterial disease in large artery ischemic stroke patients and its impact on prognosis.

\section{Methods}

This is a prospective cohort study. One hundred cases with large artery ischemic stroke were recruited from Nasr City Insurance Hospital. Patients were subjected to clinical assessment including complete medical history (detailed history including personal data with a history of risk factors including hypertension, diabetes mellitus, dyslipidemia, smoking); general examination including signs suggestive of PAD; detailed neurological history, examination, and NIHSS at days 0,7 , and 30 for the assessment of stroke severity; follow-up for recurrence or death after 6 months; laboratory assessment full chemistry including full blood picture, liver and kidney functions, fasting and random blood sugar, PT, PTT, INR, lipid profile, collagen profile, protein $\mathrm{C}$, protein $\mathrm{S}$, and anti-thrombin III in cases of stroke in young without strong risk factors; imaging including trans-thoracic echocardiography (General electric Vivid 7, USA) and trans-esophageal echocardiography in cases of stroke in young without strong risk factors to exclude cardioembolic strokes; Duplex on the arteries of both lower limbs; and measuring of ankle brachial index (ABI). The systolic pressure in the dorsalis pedis or posterior tibial artery was measured using a handheld $8-\mathrm{MHz}$ Doppler probe (model Life Dop L250R with SD8 probe product by Summit Doppler, China) and a blood pressure cuff. The higher of these two measurements was compared with a similarly taken brachial artery systolic pressure. A ratio (ankle/brachial) of 0.9 or less is considered a sign of impaired flow to the extremity [3]. MRI brain (diffusion-weighted, flair, T1, T2, and T2* images) and MRA (Philips 1.5 Tesla, Germany). Carotid duplex (General
Electric Logic 5, USA) and/or C.T angiography brain and neck and/or conventional angiography if needed.

\section{Inclusion criteria and subject selection}

Stroke patients with large artery atherosclerotic disease either intra-cranial or extra-cranial and anterior circulation or posterior circulation verified by MRI and MRA brain or CT angiography on the neck and brain vessels or conventional angiography.

\section{Exclusion criteria}

Patients were excluded from the study if they showed clinical evidence of cardio-embolic stroke as atrial fibrillation, transient ischemic attack, and intra-cerebral, subdural, or subarachnoid hemorrhage.

\section{Statistical analysis}

Data were analyzed using Stata $^{\circ}$ version 14.2 (StataCorp LLC, College Station, TX, USA). Normality of numerical data distribution was examined using the Shapiro-Wilk test. Non-normally distributed numerical data were presented as median and interquartile, and intergroup differences were compared using the Wilcoxon rank sum test (for two-group comparison) or the JonckheereTerpstra trend test (for comparison of multiple tanked grouped).

Categorical data were presented as the ratio or number and percentage, and intergroup differences were compared using Fisher's exact test (for nominal data) or the chi-squared test for trend (for ordinal data). Associations among ordinal variables were tested using the Spearman rank correlation and Kendall's tau-b.

A time-to-event analysis was done using the KaplanMeier (K-M) method. The log-rank test was used to compare individual $\mathrm{K}-\mathrm{M}$ curves.

$p$ value $<.05$ was considered statistically significant.

\section{Results}

A total of one hundred patients with acute large artery ischemic stroke were enrolled. The study included 80 males $(80 \%)$ and 20 females (20\%). The age of patients ranged from 36 to 85 with a mean of $62 \pm 9$. The most prevalent risk factor was hypertension $(80 \%)$ followed by smoking (61\%) followed by diabetes $(50 \%)$ followed by dyslipidemia (35\%) and followed by IHD (35\%), and 4\% had a history of CABG operation (Table 1). In the study group, 83 (83\%) patients had first-ever stroke while $17(17 \%)$ presented with recurrent stroke. Eighty percent of patients had anterior circulation stroke versus $14 \%$ who had post-circulation stroke while $6 \%$ had both anterior and post-circulation; among these patients, 50\% had significant extra-cranial carotid stenosis (> 50\% stenosis) and 20 cases had interventional carotid angioplasty. Among our patients, $42 \%$ had PAD (defined by 
Table 1 Characteristics of the study population

\begin{tabular}{ll}
\hline Variable & Ratio/mean \pm SD/n (\%) \\
\hline Gender (M/F) & $80 / 20$ \\
Age & $62 \pm 9$ (range, 36-85) \\
Co-morbidities & \\
DM & $50(50.0 \%)$ \\
Hypertension & $80(80.0 \%)$ \\
Dyslipidemia & $35(35.0 \%)$ \\
Smoking & $61(61.0 \%)$ \\
IHD & $35(35.0 \%)$ \\
Previous CABG & $4(4.0 \%)$ \\
\hline
\end{tabular}

$D M$ diabetes mellitus, $I H D$ ischemic heart disease, $C A B G$ coronary artery bypass graft

$\mathrm{ABI} \leq 0.9), 50 \%$ of them were asymptomatic with $\mathrm{ABI}$ range $0.6-0.9$, and $50 \%$ were symptomatic with $\mathrm{ABI}$ range 0.4-0.8. Among our 42 patients with PAD, 23 cases had ABI range from 0.7 to 0.9 , and 18 cases had marked ABI impairment below 0.7 (Fig. 1). In the study, the median for NIHSS at day 0 was 9 and interquartile range (IQR) $5-15,5$ at day 7 and IQR 3-10 while finally the median for NIHSS after 1 month was 3 and IQR 16. Eight cases out of 100 had a recurrence of large artery ischemic stroke during 1 month period, and there were 2 cases of death (Table 2). We classified the severity of PAD according to ABI to mild (0.7-0.9) and moderate to severe $(<0.7)$ [7]. There is no satisfactory significant relation between the severity of PAD and NIHSS on admission $(p$ value $=.800)$, after 7 days $(p$ value $=$ $.575)$, or at 1 month follow up ( $p$ value $=.474)$. There is no satisfactory significant relation between PAD (symptomatic or asymptomatic) and NIHSS at day $0(p$ value $=.583)$, at day $7(p$ value $=.371)$, or at day $30(p$ value $=.382)$. Five of the cases $(11.9 \%)$ who had recurrence had PAD $(\mathrm{ABI} \leq 0.9)$ versus 3
Table 2 Short-term and long-term outcomes

\begin{tabular}{ll}
\hline Variable & Median (IQR) $\mathbf{n}(\%)$ \\
\hline NIHSS & \\
Day 0 & $9(5-15)$, range $(1-23)$ \\
Day 7 & $5(3-10)$, range $(0-24)$ \\
Day 30 & $3(1-6)$, range $(0-17)$ \\
Recurrence of stroke & $8(8.0 \%)$ \\
Mortality & $2(2.0 \%)$ \\
\hline
\end{tabular}

IQR interquartile range, NIHSS National Institute of Health Stroke Scale

cases (5.2\%) without PAD (Fig. 2), with insignificant odds ratio, $\mathrm{OR}=2.47(\mathrm{CI}=95 \%), p=0.23$.

The 2 cases of mortality both had ABI $0.7-0.9$ (Figs. 2 and 3). There was no statistical significance between $\mathrm{ABI}$ and recurrence of CVS or mortality probably due to the low rate of recurrence and mortality in our study. Regarding the time to recurrence of stroke by the ABI, there is no statistically significant difference among patients with $\mathrm{ABI}>0.9, \mathrm{ABI}$ of 0.7-0.9, or $\mathrm{ABI}<0.7$ (log-rank test chi-squared = 2.293 , df $=2, p$ value $=.318$ ) (Fig. 4). The recurrence rate of stroke was higher in the PAD group with $11.9 \%$ versus $5.1 \%$ in the non-PAD group with insignificant $p$ value due to the low rate of event. Recurrence rate was higher in the asymptomatic PAD group with $19 \%$ versus $5 \%$ in the non-PAD group and 4.8 in the symptomatic PAD group. There were 2 cases of death in the asymptomatic PAD group, one at day 14 of stroke and another 6 months later with recurrence of another large artery ischemic stroke (Table 3). There is no statistically significant relation between NIHSS and recurrence of stroke with $p$ value $=0.447$ at day $0, p$ value $=$ 0.478 at day 7 , and $p$ value $=0.562$ at day 30 . Forty-eight percent of patients with significant carotid stenosis (> 50\% stenosis) has PAD versus $34 \%$ who had IHD; on the other

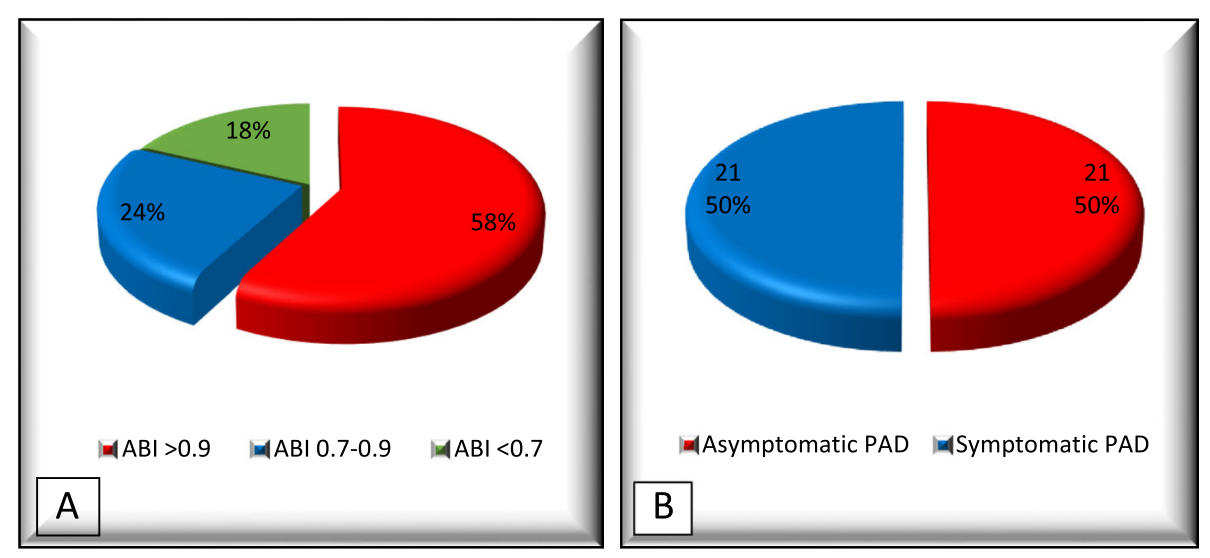

Fig. 1 a Evaluation of PAD. b Asymptomatic PAD versus symptomatic PAD 


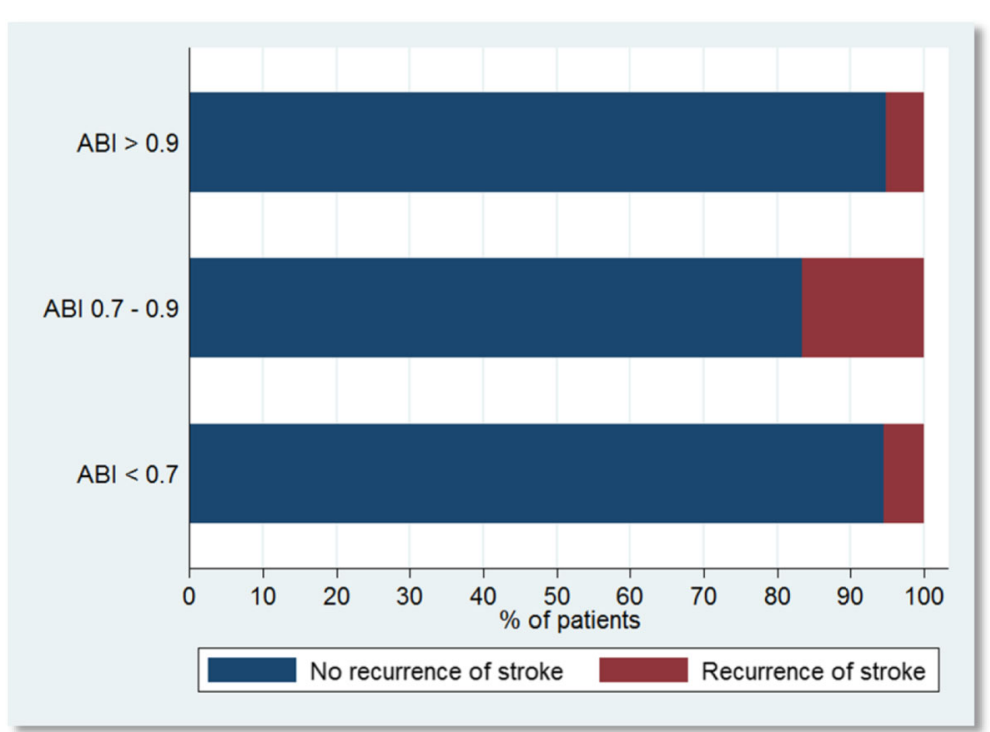

Fig. 2 Relation between $A B I$ and recurrence of stroke

hand, there was no statistically significant relation between the severity of extra-cranial stenosis and the severity of impairment of ABI (tau-p $=.099, p$ value $=0.263$ and rho $=.146)$.

\section{Discussion}

Our current study aimed to examine 100 cases with large artery ischemic stroke. The evaluation was done by history taking, clinical examination, echocardiography, MRI and MRA brain, carotid duplex or
CT angiography or conventional angiography, duplex on arteries of both lower limbs with ABI measurement, functional assessment by NIHSS at day 0,7 , and 30, and follow-up for recurrence or death after 6 months.

PAD was found in $42 \%$ of cases. This is in agreement with the Systemic Risk Score Evaluation in Ischemic Stroke Patients (SCALA) study [8] and the Polyvascular Atherothrombosis Observational Study (PATHOS) [9]. Yet, our prevalence of PAD was higher than that of a

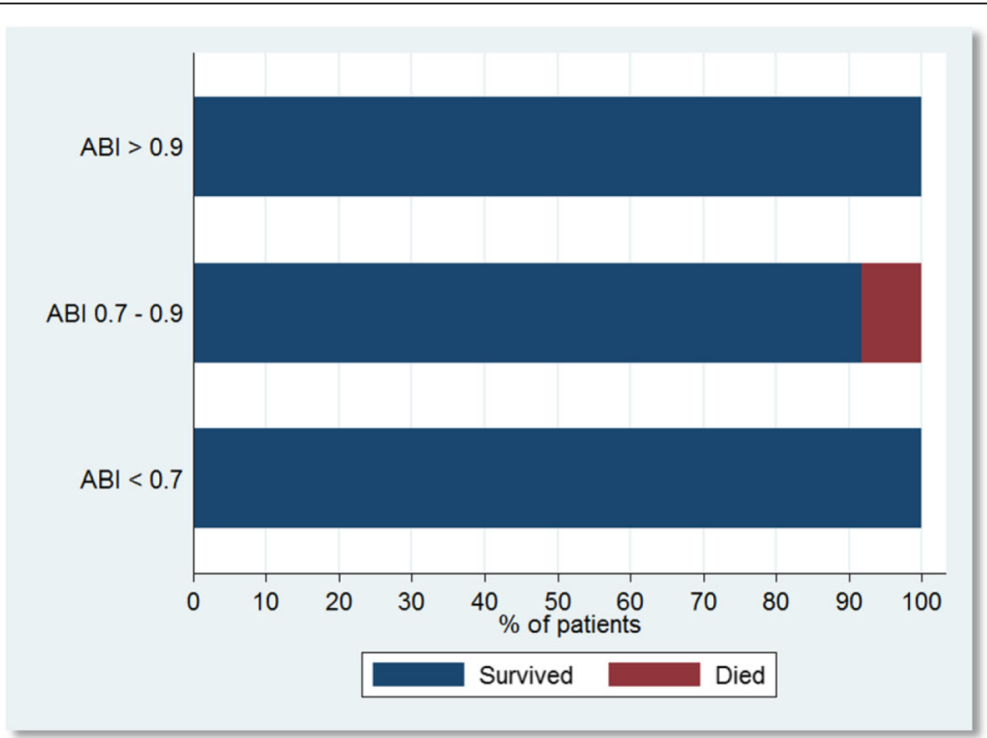

Fig. 3 Relation between $A B I$ and mortality 


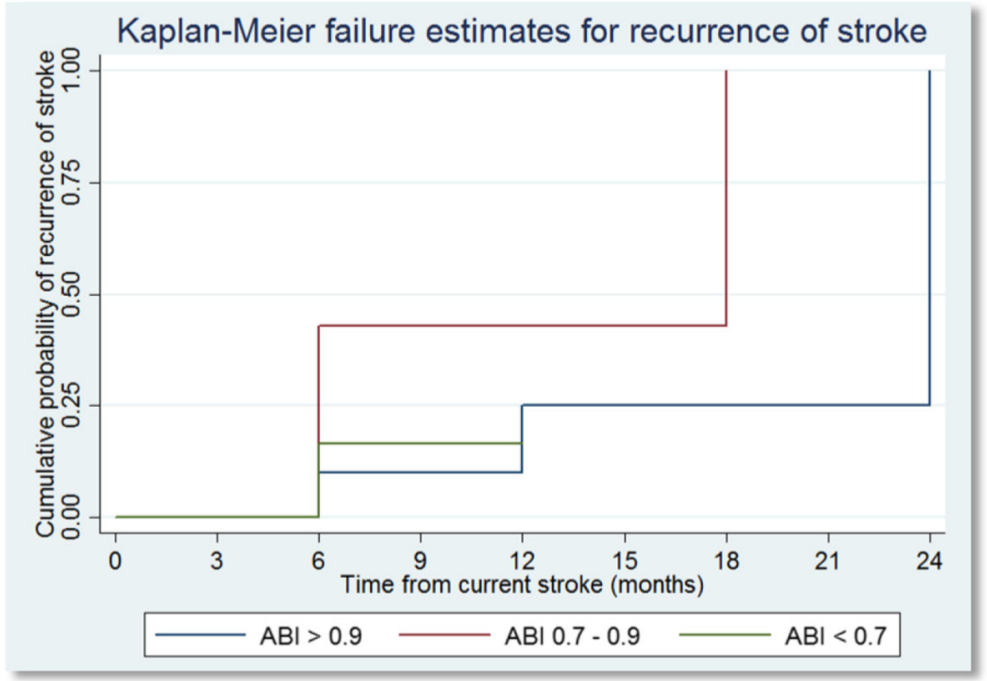

Fig. 4 Kaplan-Meier curve for the time to recurrence of stroke by the $A B I$

Japanese study, Hoshino et al. [10], indicating the possibility of ethnic variations. During our study, 8 cases had a recurrence of large artery ischemic stroke, and 5 cases $(62.5 \%)$ had PAD versus 4 cases $(50 \%)$ with IHD. This is in agreement with Uchiyama et al. [11] and Beatrice et al. [12]. Fifty percent of patients with lower extremity arterial disease were asymptomatic in agreement with Sigvant et al. [13] and Alvarez-Sabín et al. [14].

Forty-eight percent of the patients with significant carotid stenosis had PAD in agreement with another study on the Egyptian population, Sayed et al. [15], and $42 \%$ of these patients with significant carotid stenosis and PAD were asymptomatic. However, our study showed a higher percentage of significant extracranial carotid stenosis as our studied sample was only large artery ischemic stroke patients, and we studied a larger sample.

The recurrence rate of stroke was the highest in the asymptomatic PAD group with $19 \%$ followed by the non-PAD group with $5.1 \%$ followed by the symptomatic PAD group with 4.8\%; however, the low rate of recurrence in our study gives us non-significant statistical value. Notably, in the group of asymptomatic PAD patients, the risk increase was not significant, which may be due to small power owing to low event numbers. Several studies confirmed the positive association between $\mathrm{ABI}$ and stroke recurrence, yet most of them included large numbers of patients, and follow-up for recurrence and mortality was assessed at 12 and 24 months which could explain our lower events rate [16].

We strongly recommend routine screening for PAD in patients with ischemic stroke as it is a good predictor for morbidity and mortality in these patients. Once PAD is detected in ischemic stroke patients, prevention of athero-thrombtic events and treatment of atherosclerotic risk factors should be done as PAD can indicate the risk of stroke recurrence.

\section{Conclusion}

The prevalence of PAD in large artery ischemic stroke patients is high. Asymptomatic PAD has a risk potential for large artery ischemic stroke recurrence and mortality more than symptomatic PAD and non-PAD patients. $\mathrm{ABI}$ can be used in ischemic stroke patients as it is cheap, non-invasive, and available method for diagnosis of PAD for better management and prognosis of stroke patients.

Table 3 Relation between the symptoms of PAD and recurrence of stroke or mortality

\begin{tabular}{|c|c|c|c|c|c|c|c|}
\hline \multirow[t]{2}{*}{ Variable } & \multicolumn{2}{|c|}{ No PAD $(n=58)$} & \multicolumn{2}{|c|}{ Asymptomatic PAD $(n=21)$} & \multicolumn{2}{|c|}{ Symptomatic PAD $(n=21)$} & \multirow{2}{*}{$\begin{array}{l}\text { Chi-square } \\
\text { test, } p \text { value }\end{array}$} \\
\hline & $N$ & $\%$ & $N$ & $\%$ & $N$ & $\%$ & \\
\hline Recurrence of stroke & 3 & 5.1 & 4 & 19.0 & 1 & 4.8 & .663 \\
\hline Mortality & 0 & 0.0 & 2 & 9.6 & 0 & 0.0 & .515 \\
\hline
\end{tabular}




\section{Abbreviations}

ABI: Ankle-brachial index; IHD: Ischemic heart disease; PAD: Peripheral artery disease; CVD: Cerebrovascular disease; NIHSS: National Institute of Health Stroke Scale; MRA: Magnetic resonance angiography; MRI: Magnetic resonance imaging

\section{Acknowledgements}

None

\section{Authors' contributions}

SF: statistical design and execution. ME: review and critique of the manuscript. AE: idea and conception and study design. JG: data collection and research project execution. MF: writing of the first draft of the manuscript and review. All authors have agreed to the conditions noted on the Authorship Agreement Form and have read and approved the final version submitted.

\section{Funding}

No funds were received to fulfill this work.

\section{Availability of data and materials}

The corresponding author takes full responsibility for the data, the analyses and interpretation, and the conduct of the research; has full access to all of the data; and has the right to publish any and all data separate and apart from any sponsor.

\section{Ethics approval and consent to participate}

The study was approved by the Ain-Shams University Ethical Committee (date of approval: April 6, 2017) (reference number not available). Written informed consent was obtained from the patients participating in the study after informing them about the study rationale and their right to withdraw from the study at any time without any consequences.

\section{Consent for publication}

Not applicable

\section{Competing interests}

None of the authors has any conflict of interest.

\section{Author details}

${ }^{1}$ Neurology Department, Faculty of Medicine, Ain Shams University, Cairo, Egypt. ${ }^{2}$ Neurology Department, Nasr City Insurance Hospital, Cairo, Egypt.

Received: 5 June 2020 Accepted: 22 September 2020

Published online: 07 October 2020

\section{References}

1. Rothwell PM, Coull AJ, Silver LE, et al. Population-based study of event-rate, incidence, case fatality, and mortality for all acute vascular events in all arterial territories (Oxford vascular study). Lancet. 2005;366:1773-83.

2. Rutherford RB, Baker JD, Ernst C, et al. Recommended standards for reports dealing with lower limb peripheral arterial occlusion. J Vasc Surg. 1997;26: 517-38.

3. Grenon SM, Gagnon J, Hsiang Y, et al. N Engl J Med. 2009;361(19):e40. https://doi.org/10.1056/nejmvcm0807012.

4. Bhatt DL, Steg PG, Ohman EM, et al. International prevalence, recognition, and treatment of cardiovascular risk factors in outpatients with atherothrombosis. JAMA. 2006;295:180-9.

5. Hiatt WR, Hoag S, Hamman RF. Effect of diagnostic criteria on the prevalence of peripheral arterial disease. The San Luis Valley diabetes study. Circulation. 1995;91(5):1472-9.

6. Chambers BR, Donnan GA. Carotid endarterectomy for asymptomatic carotid stenosis. Cochrane Database Syst Rev. 2005:2005(4):CD001923. Published 2005 Oct 19. doi:https://doi.org/10.1002/14651858.CD001923. pub2.

7. Hirsch AT, Haskal ZJ, Hertzer NR, et al. ACC/AHA 2006 guidelines for the management of patients with peripheral arterial disease (lower extremity, renal, mesenteric, and abdominal aortic): executive summary a collaborative report from the American Association for Vascular Surgery/Society for Vascular Surgery, Society for Cardiovascular Angiography and Interventions, Society for Vascular Medicine and Biology, Society of Interventional
Radiology, and the ACC/AHA task force on practice guidelines (writing committee to develop guidelines for the Management of Patients with Peripheral Arterial Disease) endorsed by the American Association of Cardiovascular and Pulmonary Rehabilitation; National Heart, Lung, and Blood Institute; Society for Vascular Nursing; TransAtlantic inter-society consensus; and vascular Disease Foundation. J Am Coll Cardiol. 2006;47: 1239-1239.

8. Weimar C, Goertler M, Röther J, et al. Systemic risk score evaluation in ischemic stroke patients (SCALA): a prospective cross sectional study in 85 German stroke units. J Neurol. 2007;254(11):1562-8. https://doi.org/10.1007/ s00415-007-0590.

9. Agnelli G, Cimminiello C, Meneghetti G. Polyvascular Atherothrombosis observational survey (PATHOS) investigators. Low ankle-brachial index predicts an adverse 1-year outcome after acute coronary and cerebrovascular events. J Thromb Haemost. 2006:4(12):2599-606. https://doi. org/10.1111/j.1538-7836.2006.02225

10. Hoshino H, Itoh Y, Yamada S, Suzuki N. Prevalence and clinical features of asymptomatic peripheral artery disease in Japanese stroke patients. J Stroke Cerebrovasc Dis. 2013;22(3):255-9. https://doi.org/10.1016/j. jstrokecerebrovasdis.2011.08.011.

11. Uchiyama S, Goto S, Matsumoto M, et al. Cardiovascular event rates in patients with cerebrovascular disease and atherothrombosis at other vascular locations: results from 1-year outcomes in the Japanese REACH registry. J Neurol Sci. 2009:287:45-51.

12. Golomb BA, Dang TT, Criqui MH. Peripheral arterial disease morbidity and mortality implications. Circulation. 2006;114(7):688-99.

13. Sigvant B, Wiberg-Hedman $\mathrm{K}$, et al. A population-based study of peripheral arterial disease prevalence with special focus on critical limb ischemia and sex differences. J Vasc Surg. 2007:45:1185-91.

14. Alvarez-Sabín J, Gil-Núñez A, Quintana M, et al. Prevalence of asymptomatic peripheral artery disease in patients with non-cardioembolic ischemic stroke. Neurologia. 2009;24(6):366-72.

15. Sayed A, Ahmed SM, Abdelalim AM, et al. Is peripheral arterial disease associated with carotid artery disease in Egyptians? A pilot study. Egypt J Neurol Psychiatry Neurosurg. 2016;53:12-8.

16. Hong JB, Leonards CO, Endres $M$, et al. Ankle-brachial index and recurrent stroke risk meta-analysis. Stroke. 2016:47:317-22

\section{Publisher's Note}

Springer Nature remains neutral with regard to jurisdictional claims in published maps and institutional affiliations.

\section{Submit your manuscript to a SpringerOpen ${ }^{\bullet}$ journal and benefit from:}

- Convenient online submission

- Rigorous peer review

- Open access: articles freely available online

- High visibility within the field

- Retaining the copyright to your article

Submit your next manuscript at $>$ springeropen.com 